\title{
A quantitative approach to topology for fuzzy regions
}

\author{
Jörg Verstraete \\ Instytut Badań Systemowych, Polska Akademia Nauk (Systems Research Institute, \\ Polish Academy of Sciences); Ul. Newelska 6, 01-447 Warszawa, Poland \\ DDCM, Dept. Telecommunications and Information Processing, Ghent University; \\ Sint Pietersnieuwstraat 41, 9000 Ghent, Belgium \\ jorg.verstraete@\{ibspan.waw.pl, telin.ugent.be\} \\ http://www.ibspan.waw.pl,http://telin.ugent.be/ddcm

\begin{abstract}
There has been lots of research in the field of fuzzy spatial data and the topology of fuzzy spatial objects. In this contribution, an extension to the 9-intersection model is presented, to allow for the relative position of overlapping fuzzy regions to be determined. The topology will be determined by means of a new intersection matrix, and a set of numbers, expressing the similarity between the topology of the given regions and a number of predefined cases. The approach is not merely a conceptual idea, but has been built on our representation model and can as such be immediately applied.
\end{abstract}

\section{Preliminaries}

\subsection{Introduction}

A common problem in spatial reasoning, is describing the position of one object or feature in relative to another object or feature. "'Do both overlap, is one contained within the other, or do they touch?"' are some examples. For crisp regions, it is fairly easy to see that the different possibilities are mutually exclusive: if two regions touch, then one does not contain the other. The concept of a broad or undetermined boundary ([1], [2]), in which the boundary was considered to be a region delimited by an inner and an outer boundary, rather than a thin line was a first extension. The topology of such regions is similar in approach to crisp topology; all intersection cases are mutually exclusive. Allowing for truly fuzzy regions however, implies that there is no certainty or precision regarding the points of to the regions. As such, statements about topology are prone to similar uncertainty and imprecision, resulting in the fact that two regions can resemble multiple intersection cases at once (regions can for instance touch and overlap). It is important to first find the cases that match, and then to generate quantitative measures to indicate how well each matches. In this paper, we will first describe the fuzzy topology model, list some of cases, and illustrate by means of an example. 


\subsection{9-intersection model}

One approach to model crisp topology, is the 9-intersection model [5]. It uses the concepts of interior (points inside the region, denoted.$^{\circ}$ ), exterior (points the region, denoted $\cdot^{-}$) and boundary (denoted $\partial \cdot$ ), then considers every possible intersection between them. This yields a total of 9 possible intersections, commonly grouped in the matrix shown below:

$$
\left(\begin{array}{ccc}
A^{\circ} \cap B^{\circ} & A^{\circ} \cap \partial B & A^{\circ} \cap B^{-} \\
\partial A \cap B^{\circ} & \partial A \cap \partial B & \partial A \cap B^{-} \\
A^{-} \cap B^{\circ} & A^{-} \cap \partial B & A^{-} \cap B^{-}
\end{array}\right)
$$

By assigning each matrix element 0 if the intersection is empty, and 1 if the intersection is not empty, $2^{9}=512$ matrices are possible. Depending on imposed restrictions (e.g. presence of holes), only a subset of the 512 relations is possible. For crisp regions without holes and no disconnected parts in a two-dimensional space $\mathbb{R}^{2}$, only eight intersection matrices are meaningful, yielding the relations: disjoint, contains, inside, equal, meet, covers, coveredBy and overlap; illustrated on fig. 1. An alternative way of describing topology is the RCC calculus, but the nine-intersection model lends itself easier toward qualitative approach.

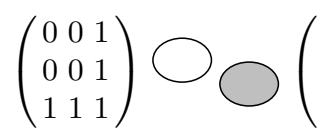

(a)

$$
\left(\begin{array}{lll}
1 & 1 & 1 \\
0 & 0 & 1 \\
0 & 0 & 1
\end{array}\right)
$$

(b)

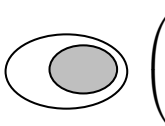

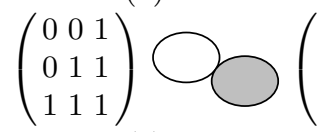

(e)

\begin{abstract}
$\left(\begin{array}{lll}1 & 1 & 1 \\ 0 & 1 & 1 \\ 0 & 0 & 1\end{array}\right)$
\end{abstract}
(f)

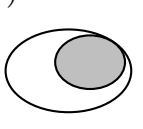

)

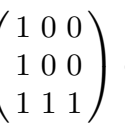

(c)

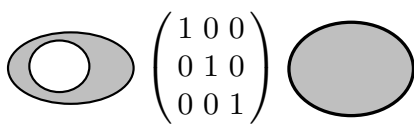

(d)

Fig. 1. Topological relations for crisp regions: disjoint (a), contains (b), inside (c), equal (d), meet (e), covers (f), coveredBy (g) and overlap (h), with their intersection matrices.

\section{$2 \quad$ Fuzzy region model}

\subsection{Concept}

Regions are often represented by means of an outline, represented by a curve. The region is defined as all the points located inside this curve ${ }^{1}$. For our definition of fuzzy regions, a different point of view is necessary: a region is considered mathematically as a set of locations (all the locations inside the curve). It then is a small step to extend it to a fuzzy set [10] of locations, where each location

\footnotetext{
${ }^{1}$ Possibly, the region can have holes, but in this contribution only regions without
} holes are considered. 
is represented by a point and each location has a membership grade associ$\operatorname{ated}^{2}$. The membership grades for regions are interpreted in a veristic way [4]: all locations belong to the region, but some more than others. A possibilistic interpretation can be used to represent fuzzy points, and although a similar approach can be applied, this contribution focusses on fuzzy regions. On fig. 2, an example of a fuzzy region is shown.

Definition 1 (fuzzy region $\tilde{A})$.

$$
\begin{gathered}
\tilde{A}=\left\{\left(p, \mu_{\tilde{A}}(p)\right) \mid p \in U, \mu_{\tilde{A}}(p)>0\right\} \\
\text { where } \\
\mu_{\tilde{A}}: U \rightarrow[0,1] \\
p \mapsto \mu_{\tilde{A}}(p)
\end{gathered}
$$

Here $U$ is the universe (commonly $\mathbb{R}^{2}$ ).

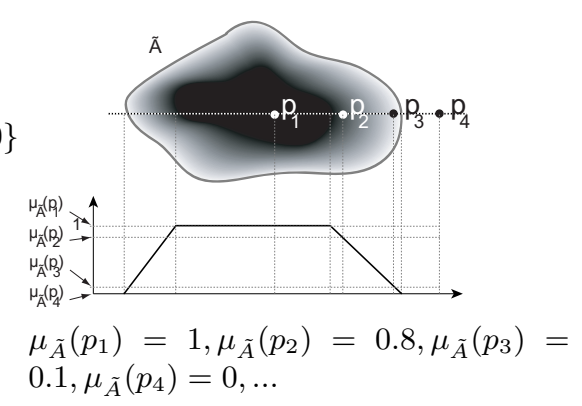

Fig. 2. A fuzzy region, for illustration purposes the fuzzy region is delimited by a grey line. The membership grades for points belonging to the region are shaded, ranging from black (membership grade 1) to white (membership grade 0). A cross section shows how the membership grades along the dotted line evolve.

\section{Topology}

\subsection{Fuzzy concepts}

To extend the 9-intersection model, appropriate definitions for interior, exterior and boundary are needed. The approach is similar to the extension in [1] for regions with broad boundaries. The definitions bear resemblance to the work of $\mathrm{Du}[3]$, where a fuzzy border around a crisp region was defined to create a fuzzy region. This is similar to our work in [7], but with such regions it is impossible to make a closed calculus: the intersection for instance cannot always be represented by a new such region. For the fuzzy boundary, several consideration are in place: as the region itself is fuzzy, it is logical that its boundary will be a fuzzy entity. It should be defined such that it remains compatible with the crisp topology. First, consider the case of a fuzzy region which has a membership grade 1 in some central part, and has continuously, decreasing membership grades away from this central part outward. Points $p$ with membership grade $\mu_{\tilde{A}}(p)=0$ or $\mu_{\tilde{A}}(p)=1$ in the original region can be considered not to belong to the boundary, as they are completely outside, respectively completely inside the region. Points with a

\footnotetext{
${ }^{2}$ Note that the membership grade are not derived from coordinates of the point, but assigned which each point individually.
} 
membership grade that is closer to 0 or 1 belong less to the boundary than points with a membership grade closer to 0.5 . Because of this 0.5 will play a crucial part: points $p$ for which $\mu_{\tilde{A}}(p)=0.5$ will be said to completely belong to the boundary (and thus $\mu_{\Delta \tilde{A}}(p)=1$ ). The more $\mu_{\tilde{A}}(p)$ differs from 0.5 , the lower $\mu_{\Delta \tilde{A}}(p)$ should be. This can be accomplished with e.g. the function: $2(0.5-|0.5-x|), \forall x \in[0,1]$; illustrated on fig. 3a, the resulting boundary is shown on fig. 3b and fig. 3c. The particular function was chosen as it it keeps some properties of the original function when only considering the values in $[0,0.5]$ or $[0.5,1]$ (e.g. linearity). As crisp regions (and broad boundary regions) are special cases of fuzzy regions, the definition must be such that remains compatible (this is also required if for instance a fuzzy region's has a sudden transition of membership grade at some part). This is achieved by considering the boundaries $\partial \tilde{A}_{\alpha}$ at every $\alpha$-level $\alpha$. The definition of the boundary of a fuzzy region $\tilde{A}$ is given in fig. 3 . In general, it is not mandatory to have points with a membership grade 0.5 . For a region it may therefore be possible that at some sides there exists a path from the core to the outside which does not cross points for which $\mu_{\Delta \tilde{A}}(p)=1$ (the current definition guarantees there always will be points for which $\mu_{\Delta \tilde{A}}(p)>0$ that will be crossed). The full impact of this is currently under investigation. For the interior, first consider the points that are completely part of the region

Definition 2 (boundary $\Delta \tilde{A}$ ). $\Delta \tilde{A}=\left\{\left(p, \max \left(\sup \left\{\alpha \mid p \in \partial \tilde{A}_{\alpha}\right.\right.\right.\right.$, $\left.\left.2\left(0.5-\left|0.5-\mu_{\tilde{A}}(p)\right|\right)\right)\right\}$

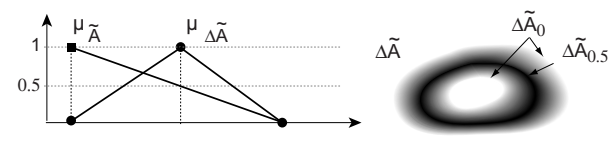

Definition 3 (interior $\tilde{A}^{\circ}$ ).

$$
\begin{aligned}
\tilde{A}^{\circ}=\left\{\left(p, \mu_{\tilde{A}^{\circ}}(p)\right\}\right. \text { where } & \\
\mu_{\tilde{A}^{\circ}}: U & \rightarrow[0,1] \\
p & \mapsto \begin{cases}0 & \mu_{\tilde{A}}(p) \leq 0.5 \\
1-\mu_{\Delta \tilde{A}}(p) & \text { elsewhere }\end{cases}
\end{aligned}
$$
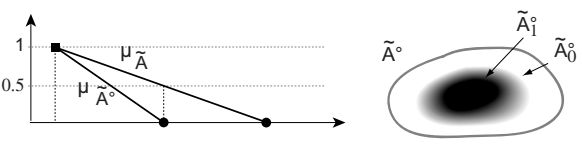

$$
\begin{aligned}
& \text { Definition } 4 \text { (exterior } \tilde{A}^{-} \text {). } \\
& \tilde{A}^{-}=\left\{\left(p, \mu_{\tilde{A}^{-}}(p)\right\}\right. \text { where } \\
& \mu_{\tilde{A}^{-}}: U \rightarrow[0,1] \\
& p \mapsto \begin{cases}0 & \mu_{\tilde{A}}(p) \geq 0.5 \\
1-\mu_{\Delta \tilde{A}}(p) & \text { elsewhere }\end{cases}
\end{aligned}
$$
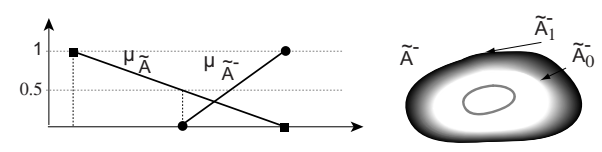

Fig. 3. Illustration of the fuzzy boundary, interior and exterior; for each, the definition, a plot of the membership function (with the membership function for $\tilde{A}$ ) and a graphical representation of the resulting regions using grey scales.

$\left(\mu_{\tilde{A}}(p)=1\right)$; this will also be the core of the interior. Points $p$ just outside this core, but still belonging to a substantial extent to the region (i.e. $\mu_{\tilde{A}}(p)>0.5$ ) are also considered to be part of the interior to a lesser extent. Points $p$ with a 
membership grade $\mu_{\tilde{A}}(p) \leq 0.5$, are considered not to belong to the interior, fig. 3 . The exterior is defined analogously, fig. 3 .

\subsection{Intersection matrices}

Description Once the concepts of interior, boundary and exterior are known, the 9-intersection matrix for fuzzy regions is:

$$
\left(\begin{array}{ccc}
h\left(\tilde{A}^{\circ} \tilde{\tilde{n}} \tilde{B}^{\circ}\right) & h\left(\tilde{A}^{\circ} \tilde{\tilde{n}} \Delta \tilde{B}\right) & h\left(\tilde{A}^{\circ} \tilde{\tilde{n}} \tilde{B}^{-}\right) \\
h\left(\Delta \tilde{A} \tilde{\cap} \tilde{B}^{\circ}\right) & h(\Delta \tilde{A} \tilde{\cap} \Delta \tilde{B}) & h\left(\Delta \tilde{A} \tilde{\cap} \tilde{B}^{-}\right) \\
h\left(\tilde{A}^{-} \tilde{\cap} \tilde{B}^{\circ}\right) & h\left(\tilde{A}^{-} \tilde{\cap} \Delta \tilde{B}\right) & h\left(\tilde{A}^{-} \tilde{\cap} \tilde{B}^{-}\right)
\end{array}\right)=\left(\begin{array}{ccc}
d & c_{1} & e_{1} \\
c_{2} & b & a_{1} \\
e_{2} & a_{2} & 1
\end{array}\right)
$$

Here $h(X)$ is the notation for the height $(X)$ of a fuzzy set $X$, i.e. the highest membership grade in the set [6]. The intersection is the fuzzy intersection, by means of a t-norm (e.g. minimum). The matrix elements can have any value in the range $[0,1]$, which impacts how the matrices will be interpreted; and are named (apart from the bottom right element, which is always 1). A full case study has been made, yielding a large number of cases; but as there are similarities there are ways of grouping them. We opted for groups more or less resembling the 44 cases Clementini listed for the broad boundary model ([1]). Two cases are illustrated below, their numbers referring to their number in the 44 cases.

- Case 1: The first case is when both regions $\tilde{A}$ and $\tilde{B}$ are completely disjoint.

$$
\tilde{A} \text { disjoint } \tilde{B} \widetilde{A} \bigcirc{ }^{\tilde{B}}\left(\begin{array}{ccc}
0 & 0 & 1 \\
0 & 0 & a_{1} \\
1 & a_{2} & 1
\end{array}\right) \text { with }\left\{\begin{array}{l}
\left.\left.a_{1}, a_{2} \in\right] 0,1\right] \\
b, d, c_{1}, c_{2}=0 \\
e_{1}, e_{2}=1
\end{array}\right.
$$

This matrix is similar to the nine-intersection matrix of disjoint crisp regions, and to the nine-intersection matrix of disjoint regions with broad boundaries. The main difference is that the elements $a_{1}$ and $a_{2}$ are both in the range ]0,1], rather than 1 . We can only be sure that it they equal 1 if there are elements in the region with membership grade 0.5 (membership grade in the boundary is then 1). While this is the case in this assumption, we cannot be sure of this in general.

- Case 3 occurs when the boundary $\Delta \tilde{A}$ intersects with the boundary $\Delta \tilde{B}$ and when the interior $\tilde{A}^{\circ}$ also intersects with the boundary $\Delta \tilde{B}$ (or vice versa: case 6 ). An intersection between the boundaries implies that $h(\Delta \tilde{A} \tilde{\cap} \Delta \tilde{B})>0$. It is possible for this matrix element to equal 1 , when there are points for which $\mu_{\tilde{A}}(p)=\mu_{\tilde{B}}(p)=$ 0.5 . Second, it is possible for $\Delta \tilde{A}$ to intersect with $\tilde{B}^{\circ}$. Even further, if the interior of $\tilde{A}$ intersects with the boundary of $\tilde{B}$, this means that $h\left(\tilde{A}^{\circ} \tilde{\cap} \Delta \tilde{B}\right)>0$. It is possible for this element to equal 1 , if there are points $p$ such that $\mu_{\tilde{A}}(p)=1 \wedge \mu_{\tilde{B}}(p)=0.5$; but it is not possible for this element to equal 0 (as this would yield a different case).

$$
\begin{aligned}
& \begin{array}{l}
\Delta \tilde{A} \tilde{\cap} \tilde{B}^{\circ}=\emptyset \tilde{A} \\
\tilde{A}^{\circ} \tilde{\cap} \tilde{B}^{\circ}=\emptyset
\end{array} \\
& \begin{array}{l}
\Delta \tilde{A} \tilde{\cap} \tilde{B}^{\circ} \neq \emptyset \tilde{A} \\
\tilde{A}^{\circ} \tilde{\cap} \tilde{B}^{\circ}=\emptyset
\end{array} \quad{ }^{\tilde{B}}\left(\begin{array}{ccc}
0 & c_{1} & 1 \\
c_{2} & b & a_{1} \\
1 & a_{2} & 1
\end{array}\right) \text { with }\left\{\begin{array}{l}
\left.\left.a_{1}, a_{2}, b, c_{1} \in\right] 0,1\right] \\
\left.c_{2} \in\right] 0,1[
\end{array}\right. \\
& \begin{array}{l}
\Delta \tilde{A} \tilde{\cap} \tilde{B}^{\circ} \neq \emptyset \tilde{\mathrm{A}} \\
\tilde{A}^{\circ} \tilde{\cap} \tilde{B}^{\circ} \neq \emptyset
\end{array} \quad \widetilde{B}\left(\begin{array}{ccc}
d & c_{1} & 1 \\
c_{2} & b & a_{1} \\
1 & a_{2} & 1
\end{array}\right) \text { with }\left\{\begin{array}{l}
\left.\left.a_{1}, a_{2}, b, c_{1} \in\right] 0,1\right] \\
\left.c_{2}, d \in\right] 0,1[
\end{array}\right.
\end{aligned}
$$


The above matrices can be combined: $\left(\begin{array}{ccc}d & c_{1} & 1 \\ c_{2} & b & a_{1} \\ 1 & a_{2} & 1\end{array}\right)$ with $\left\{\begin{array}{l}\left.\left.a_{1}, a_{2}, b, c_{1} \in\right] 0,1\right] \\ \left.c_{2} \in\right] 0,1[ \\ d \in[0,1[\end{array}\right.$

Other cases Due to lack of space, we cannot list all the cases in this contribution. To help with the example, we will only list the conditions on the matrix elements for cases 4,5,10 and 11 .

- case 4: the boundary $\Delta \tilde{A}$ intersects with the boundary $\Delta \tilde{B}$ and the interior $\tilde{A}^{\circ}$ is entirely located inside the broad boundary $\Delta \tilde{B}$ (case 7 is the symmetrical case); $\left.\left.\left.\left.\left.\left.\left.\left.a_{1} \in\right] 0,1\right], a_{2} \in\right] 0,1\right], b \in\right] 0,1\right], c_{1} \in\right] 0,1\right], c_{2} \in\left[0,1\left[, d \in\left[0,1\left[, e_{1} \in\left[0,1\left[, e_{2}=1\right.\right.\right.\right.\right.\right.$.

- case 5: the boundary $\Delta \tilde{A}$ and a fortiori the interior $\tilde{A}^{\circ}$ are completely inside the boundary $\Delta \tilde{B}$ (case 8 is the symmetrical case); $\left.\left.a_{1} \in\left[0,1\left[, a_{2} \in\right] 0,1\right], b \in\right] 0,1\right]$, $\left.\left.c_{1} \in\right] 0,1\right], c_{2} \in\left[0,1\left[, d \in\left[0,1\left[, e_{1} \in\left[0,1\left[, e_{2}=1\right.\right.\right.\right.\right.\right.$.

- case 10: the boundary $\Delta \tilde{A}$ intersects with the interior $\tilde{B}^{\circ}$, the boundary $\Delta \tilde{B}$ and the exterior $\tilde{B}^{-}$. The interior $\tilde{A}^{\circ}$ can intersect with either $\Delta \tilde{B}$ (to a degree of up to 1 ) and with $\tilde{B}^{\circ}, \tilde{B}^{-}$(to a degree strictly less than 1$\left.\left.\left.\left.\left.\left.) ; a_{1} \in\right] 0,1\right], a_{2} \in\right] 0,1\right], b \in\right] 0,1\right]$, $\left.\left.\left.\left.c_{1} \in\right] 0,1\right], c_{2} \in\right] 0,1\right], d \in\left[0,1\left[, e_{1} \in\left[0,1\left[, e_{2}=1\right.\right.\right.\right.$.

- case 11: the boundary $\Delta \tilde{A}$ intersects with the interior $\tilde{B}^{\circ}$ and the boundary $\Delta \tilde{B}$. An intersection with the exterior $\tilde{B}^{-}$is possible, but only to a degree strictly less than 1 . The interior $\tilde{A}^{\circ}$ can intersect with either $\tilde{B}^{\circ}, \Delta \tilde{B}$ and $\tilde{B}^{-} ; a_{1} \in[0,1[$, $\left.\left.\left.\left.\left.\left.\left.\left.a_{2} \in\right] 0,1\right], b \in\right] 0,1\right], c_{1} \in\right] 0,1\right], c_{2} \in\right] 0,1\right], d \in\left[0,1\left[, e_{1} \in\left[0,1\left[, e_{2} \in[0,1[\right.\right.\right.\right.$

In the conceptual neighbourhood graph (a graph where two cases are considered neighbours and thus connected when the changes between them are as small as possible), cases 4 and 11 are connected with cases 5 and 10 .

Interpretation When grouped in 44 cases, each matrix element is restricted to one of the following intervals: $[0],[0,1[] 0,1],,[1]$. These can be said to have an intuitive order: [0,1[ can said to be smaller than ]0,1] because the largest possible value is smaller; ]0,1] smaller than 1 as smaller values are possible, and similarly 0 is smaller than [0,1[. The case matrices can be grouped according to each element restriction, and a conceptual neighbourhood graph can be constructed. For two regions, their intersection matrix will contain 9 values in the range $[0,1]$; for each value the matching cases are sought: if the value satisfies the element constraint, the case is retained. After this, the number of matching cases will be reduced (not necessarily to 1); and they will be neighbours in the conceptual neighbourhood graph. A number will determine how well the each one matches with the given matrix. To find this number, we apply the rule that values smaller than 0.5 belong more $[0,1[$ than to $] 0,1]$; whereas values greater than 0.5 have the opposite property (this is an intuitive rule, the value 0.5 is present in both intervals). Now, match values are assigned for every matrix element $x$ that distinguishes two groups and for every case $i$ that is in either of the two groups. These match values represent how well each matrix element matches; aggregating them for a case $i$ (using a t-norm), yields a single value expressing how well the given matrix matches this case.

Definition 5 (Match value $m_{x}^{i}$ for a case $i$ and a matrix element $x$ ).

$$
m_{x}^{i}=\left\{\begin{array}{c}
x \quad \text { if range of case } i=] 0,1] \\
1-x \text { if range of case } i=[0,1[
\end{array}\right.
$$




\subsection{Example}

To illustrate, consider two regions that yield the following intersection matrix:

$$
\left(\begin{array}{ccc}
d & c_{1} & e_{1} \\
c_{2} & b & a_{1} \\
e_{2} & a_{2} & 1
\end{array}\right)=\left(\begin{array}{ccc}
0.6 & 0.7 & 0.4 \\
0.4 & 0.6 & 0.3 \\
1 & 0.6 & 1
\end{array}\right)
$$

This example will be examined in further detail.

- $a_{1}$ and $a_{2}$ : For the element $a_{1}$, there are only two groups: $\left.] 0,1\right]$ and $[0,1[$, and both groups are possible for the given value. We come to the same conclusion for $a_{2}$. The possible cases with these values are all intersection cases.

$-b$ : For the value of $b$, there are three groups: 0,$] 0,1]$ and $[0,1[$. Obviously, the value for $b$ is not 0 , so the cases with $b=0$ (only case 1 ) are not possible.

$-c_{1}$ and $c_{2}$ : For the value of $c_{1}$, there are three groups: 0,$\left.] 0,1\right]$ and $[0,1[$. The cases with $c_{1}=0$ (cases 1 and 39) will not match our given matrix. Similarly, cases 1 and 40 don't match with the value of $c_{2}$.

$-d$ :For the value $d$, there are three groups: $0,[0,1[$ and 1 . In the example, $d=0.6$; only the cases with $[0,1[$ remain: $\{2,3,4,5,6,7,8,9,10,11,12,13,14,17\}$

- $e_{1}$ and $e_{2}$ : For $e_{1}$, there are three groups: 0, [0,1[ and 1 ; for $e_{1}=0.4$, only [0,1[ is applicable, leaving: $\{4,5,10,11,14,17,19,20,23,24,25,26,29,30,32,35,36,38,41,42$, $43,44\}$. For $e_{2}=1$ it is similar, but now the cases for which $e_{2}=1$ is required. This leaves: $\{1,2,3,4,5,6,9,10,11,18,19,20,27,28,39\}$

The intersection of all of the above cases results in the cases which are appropriate for the example: $\{4,5,10,11\}$. The differences between the cases $4,5,10$ and 11 is in the elements that match with the values of $a_{1}=0.3, c_{1}=0.7$ and $c_{2}=0.4$. To find the closest match, we need the different match values.

$$
\begin{array}{cl}
a_{1}=0.3 \text { cases 4,10: } & 0<a_{1} \leq 1 \Rightarrow m_{a_{1}}^{4}=m_{a_{1}}^{10}=0.3 \\
\text { cases 5,11: } & 0 \leq a_{1}<1 \Rightarrow m_{a_{1}}^{5}=m_{a_{1}}^{11}=0.7 \\
c_{1}=0.7 \text { case 5: } & 0 \leq c_{1}<1 \Rightarrow m_{c_{1}}^{5}=0.3 \\
\text { cases 4,10,11: } & 0<c_{1} \leq 1 \Rightarrow m_{c_{1}}^{4}=m_{c_{1}}^{10}=m_{c_{1}}^{11}=0.7 \\
c_{2}=0.4 \operatorname{cases~4,5:~} & 0 \leq c_{2}<1 \Rightarrow m_{c_{2}}^{4}=m_{c_{2}}^{5}=0.6 \\
\text { cases 10,11 } & 0<c_{2} \leq 1 \Rightarrow m_{c_{2}}^{10}=m_{c_{2}}^{11}=0.4
\end{array}
$$

The match values for $a_{1}$ indicate that cases 5 and 11 are a better match for the example than cases 4 and 10 . The match values for $c_{1}$, show that cases $4,10,11$ are a better match than case 5 ; according to $c_{2}$ cases 4 and 5 are a better match than cases 10 and 11 . The aggregation of the match values yields:

$$
\begin{aligned}
\text { case } 4: \min \left\{m_{a_{1}}^{4}, m_{c_{1}}^{4}, m_{c_{2}}^{4}\right\} & =\min \{0.3,0.7,0.6\}=0.3 \\
\text { case } 5: \min \left\{m_{a_{1}}^{5}, m_{c_{1}}^{5}, m_{c_{2}}^{5}\right\} & =\min \{0.7,0.3,0.6\}=0.3 \\
\text { case } 10: \min \left\{m_{a_{1}}^{10}, m_{c_{1}}^{10}, m_{c_{2}}^{10}\right\} & =\min \{0.3,0.7,0.4\}=0.3 \\
\text { case } 11: \min \left\{m_{a_{1}}^{11}, m_{c_{1}}^{11}, m_{c_{2}}^{11}\right\} & =\min \{0.7,0.7,0.4\}=0.4
\end{aligned}
$$

As the aggregated match value is the highest for case 11, the topology for the example is closer to this case than to any of the other three cases. However, the differences between the aggregated match values are very small, so the regions in the example still resemble the other three cases quite closely. 


\section{Conclusion}

In this paper, we presented a qualitative approach for judging the topology of fuzzy regions, represented by our model. The approach yields a soft classification in which predefined cases are matched and a degree of this match is provided. The topology is a generalization of Clementini's broad boundary model: the fuzzy region model can be used to represent broad regions, and if all points inside the inner region are assigned membership grade 1, all points of the broad boundary are assigned a membership grade 0.5 , and all points outside of the outer boundary membership grade 0 , the topology cases and matrices match. It also generalizes the topology for crisp regions: assigning points inside the region the membership grade 1 , and points outside the crisp region membership grade 0 , the fuzzy methodology results in the classical 9-intersection model. The methodology has been illustrated using our theoretical model, but is equally applicable on the models for implementation purposes we derived from this model ([8],[9]).

\section{References}

1. Clementini E., Di Felice P., An algebraic model for spatial objects with undetermined boundaries, In: GISDATA Specialist Meeting - revised version (1994)

2. Cohn A. G., Gotts N. M., Spatial regions with undetermined boundaries, In: Proceedings of the Second ACM Workshop on Advances in GIS, 52-59 (1994)

3. Du S., Qin Q., Wang Q., Li B., Fuzzy description of topological relations I: a unified fuzzy 9-Intersection model, In: Advances in Natural Computation, LNCS, vol. 3612, Lipo Wang, Ke Chen and Yew Soon Ong (Eds.), 12611273 (2005)

4. Dubois D., Prade H.: Fundamentals of Fuzzy Sets. Kluwer Academic Pub. (2000)

5. Egenhofer M.J., Sharma J., Topological Relations Between Regions in $R^{2}$ and $Z^{2}$, In: Advances in Spatial Databases - Third International Symposium SSD'93, D. Abel and B.C. Ooi (Ed.), LNCS 692, Springer-Verlag, Singapore, 316-336, (1993)

6. Klir G. J., Yuan B.: Fuzzy sets and fuzzy logic: Theory and applications. New Jersey: Prentice Hall, (1995)

7. Verstraete J., Van der Cruyssen B., De Caluwe R., Assigning Membership Degrees to Points of Fuzzy Boundaries, In: NAFIPS2000 Conf. Proc., Atlanta, 444-447, (2000)

8. Verstraete J., De Tré G., Hallez A.: Adapting TIN-layers to Represent Fuzzy Geographic Information. In: The Seventh Meeting of the EURO Working Group on Fuzzy Sets, pp. 57-62 (2002)

9. Verstraete J., Hallez A., De Tré G.: Bitmap Based Structures for the modelling of Fuzzy Entities. Special issue of Control \& Cybernetics vol. 35 no. 1, 147-164, (2006)

10. Zadeh L.A.: Fuzzy Sets. Information and Control 1 3, 338-353 (1965) 